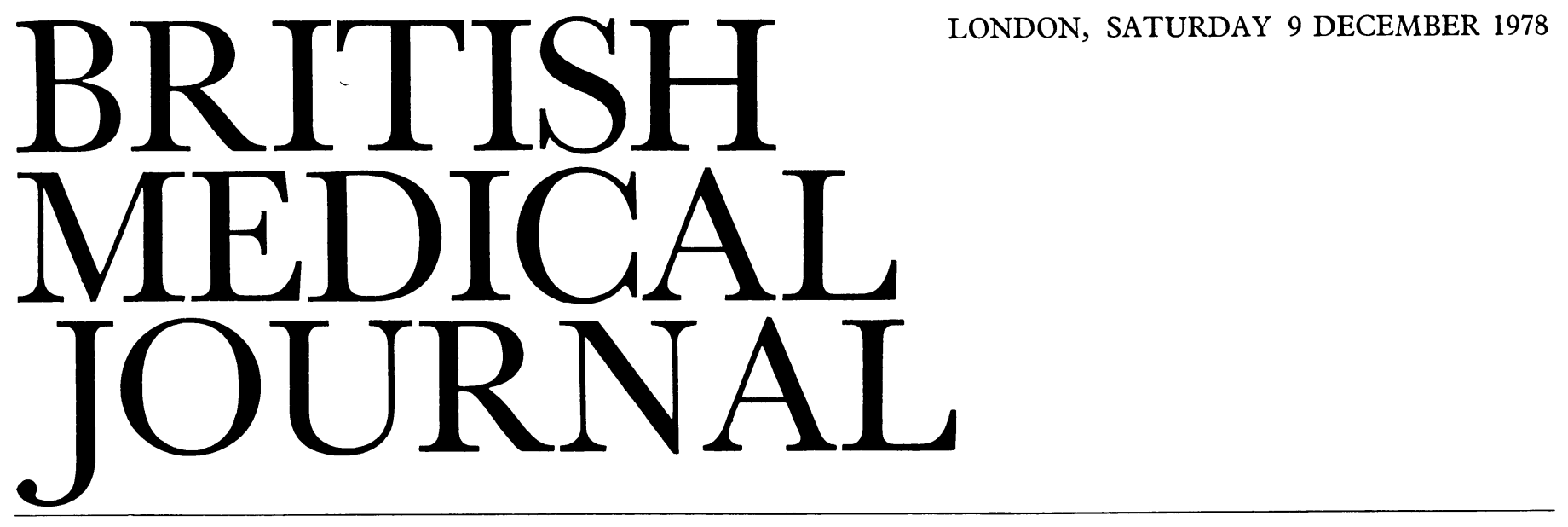

\title{
Clofibrate and the primary prevention of ischaemic heart disease
}

Just how little we know about the primary prevention of ischaemic heart disease is shown by the controversy that still surrounds the use of diet and drugs in its prevention. Some have heavily criticised primary intervention on these lines ${ }^{1}$ while others have vigorously defended it, even after myocardial infarction, claiming that an overall reduction of the serum cholesterol concentration by $10-20^{\circ}$ o (by use of diet or drugs, or both) could hypothetically cause a $6 \%$ decrease in the death rate. ${ }^{2}$

The recent report of a multicentre trial of clofibrate in the primary prevention of ischaemic heart disease is therefore timely and makes complex but valuable reading. ${ }^{3}$ The first reports $^{45}$ of the use of clofibrate as a lipid-lowering drug appeared in 1962. Later it was shown to decrease the concentration of the serum triglyceride more than that of the cholesterol. ${ }^{6}$ The present paper reports a prospective trial in healthy volunteers designed to see whether lowering the plasma lipid concentrations with clofibrate alone would diminish the incidence of ischaemic heart disease. The trial was carried out in Edinburgh, Budapest, and Prague on a total of $15745 \mathrm{men}$, aged between 30 and 59 years at the time of entry, who were treated for an average of 5.3 years. The first treatment group consisted of a randomly chosen half of the men whose serum cholesterol concentrations fell within the upper third of the distribution in some 30000 volunteers who were tested. The other 5000 men with the same cholesterol concentrations received placebo. A second control group comprised 5000 men selected at random from the lower third of the cholesterol distribution. Such large numbers were needed so that the research group could be $90 \%$ certain that they could detect a $30 \%$ reduction in the incidence of ischaemic heart disease, should this be achieved. The planned reduction in plasma cholesterol concentrations in the treatment group was $15^{\circ} \%$; in fact, the mean decrease was only $9^{\circ}$. This same amount of reduction might have been achieved by changing the dict. Nevertheless, the reduction in the serum triglyceride concentrations was greater, though these studies were carried out only in Edinburgh and without baseline values; the postulated importance of triglycerides as an independent risk factor ${ }^{7}$ remains uncertain.

What, then, of the results, if the reduction in plasma cholesterol concentration was relatively unimpressive? Firstly, those who believe that a low concentration is associated with a lower incidence of ischaemic heart disease and that control of hypercholesterolaemia is essential to the long-range prevention of atherosclerotic disease ${ }^{8}$ will draw encouragement from the fact that the control group within the lower third of the cholesterol distribution had substantially lower rates of ischaemic heart disease than either of the other two groups. In the groups with the higher range of cholesterol concentrations, clofibrate made no difference to the incidence of fatal heart attacks, nor was there a significant difference in the incidence of angina. On the other hand, there was a definite reduction in the incidence of non-fatal myocardial infarcts $\left(25^{\circ}\right)$ in those who received clofibrate, producing an overall lowering of the incidence of ischaemic heart disease by $20 \%$ in the clofibrate group as compared with the controls with high cholesterol concentrations. This reduction in myocardial infarction was greatest in men with the highest concentrations of cholesterol treated with clofibrate-the group that also showed the greatest reduction in their serum cholesterol concentrations.

Thus clofibrate has some value: a reduction of the incidence of myocardial infarcts, even with no change in the mortality rate, may well have social benefits. These results do, however, leave unanswered the question why there should be a reduction in non-fatal as opposed to fatal infarcts-one that cannot necessarily be answered by epidemiological studies but that may require reassessment of possible associated effects of clofibrate. This is not the first time that questions have remained unanswered after careful study of the clinical use of clofibrate: similar problems occurred with the Scottish and Newcastle trials in patients with angina. ${ }^{9}$ Whether or not clofibrate may slow down the development of coronary atherosclerosis has yet to be tested in clinical trial.

While this careful and comprehensive study showed as the principal benefit a reduction in the incidence of non-fatal myocardial infarcts, the overall impact of clofibrate on death and illness in these 15000 individuals was, unfortunately, apparently adverse. Both the number of deaths and the crude mortality rates from all causes in the clofibrate-treated group were significantly higher than those in the control group with high cholesterol concentrations, even though the agestandardised mortality rates did not differ significantly among the three groups. Furthermore the incidence of gall stones requiring cholecystectomy was much greater in the clofibrate group than in the other two $(P<0.001)$. Perhaps the lower incidence of deaths due to disease affecting the liver, biliary tract, and intestinal system in the controls with high cholesterol concentrations than in either of the other two groups was due 
to chance, but the higher rate in the clofibrate-treated group cannot be ignored.

What conclusions can we draw from this detailed study of so many patients for so long? The authors believe that the reduced incidence of non-fatal ischaemic heart disease in the treated patients was attributable to the modest reduction they achieved in serum cholesterol concentrations. In some quarters $^{2}$ that might be seen as justification for arguing that a greater reduction in the serum cholesterol concentration might prove more beneficial-and so might lead to calls for trials of other substances, such as cholestyramine, ${ }^{6}$ with a greater lipid-lowering potential. Research trials would, however, encounter serious problems of compliance and the selection of placebo. Furthermore, the clofibrate trials have raised several awkward possibilities. There may well prove to be metabolic effects of its long-term use that are as yet unrecognised: the authors acknowledge (as is implicit in their data) that the drug may have serious pathological consequences by mechanisms as yet unexplained.

The immediate practical conclusion must be that clofibrate can no longer be recommended as a lipid-lowering agent for general use (though it may still have a place in treating specific hyperlipidaemias). Furthermore, since there are so many unanswered questions, the other lipid-lowering regimens recommended for general use may possibly share the same problems: and regimens of this kind-including, perhaps, dietary modification with polyunsaturated fats-will require equally careful consideration in the future if we are to achieve benefit without at the same time causing more harm than had individuals been left untreated.

\footnotetext{
${ }^{1}$ McMichael, J, European fournal of Cardiology, 1977, 5, 447.

2 Coronary Drug Project Research Group, American fournal of Cardiology, 1978, 42, 489.

${ }^{3}$ Committee of Principal Investigators, British Heart fournal, 1978, 40, 1069.

${ }^{4}$ Oliver, M F, Lancet, 1962, 1, 1321.

5 Thorp, J M, Lancet, 1962, 1, 1323.

${ }^{6}$ Stone, N J, Annals of Internal Medicine, 1972, 77, 280.

7 Carlson, L A, and Böttiger, L E, Lancet, 1972, 1, 865.

${ }^{8}$ Mott, G E, McMahan, C A, and McGill, H C, jun, Circulation Research, $1978,43,364$

${ }^{9}$ Dewar, H A, and Oliver, M F, British Medical Fournal, 1971, 4, 784.
}

\section{Is hyperthermia a teratogen?}

Many studies in different species of animals have shown that induction of maternal hyperthermia can have a teratogenic effect on the fetus. ${ }^{1-6}$ Nevertheless, we still do not know whether human congenital malformations ever have this cause. Hyperthermia does occur in pregnancy-as a result of an acute infection or of an external stimulus such as a sauna bath $^{7}$ or even perhaps a heat wave.

The possibility that that most common of febrile illnesses, influenza, might cause malformations of the central nervous system has been considered ever since De Lee ${ }^{8}$ had noted that after the 1918 pandemic a number of "monstrosities" had been delivered to women in his practice. The Asian influenza pandemic of 1957-8 prompted many prospective and retrospective studies into its possibly deleterious effects on the fetus. ${ }^{9-19}$ The conclusion was best summed up by Doll: ${ }^{11}$ that Asian influenza contracted at the susceptible period of gestation might well increase the risk of anencephaly, but probably only by a factor of 2 . Such an effect would therefore be unlikely to be detected in most series of cases.
Recently, Miller, Smith, and Shephard ${ }^{20}$ retrospectively questioned 63 women who had had anencephalics in the previous 10 years to ascertain whether they had had periods of hyperthermia during pregnancy. Five women reported a febrile illness at 5-6 weeks after their last menstrual period and a further two had had saunas at this time. Control women reported no such history. As Leck ${ }^{21}$ has pointed out, however, data from a retrospective inquiry are always prone to bias. $\mathrm{He}$ also noted that in Finland, where pregnant women regularly take saunas, the incidence of anencephaly is one of the lowest in the world.

A different type of study from the Dysmorphology Unit of the University of Washington ${ }^{22}$ looked at 915 patients with "multiple defects of unknown aetiology." Among the questions put to the mothers was one on febrile illness in early pregnancy. Eight cases gave such a history between six and eight weeks from the date of the last menstrual period. These eight cases had the stigmata of a syndrome which included mental deficiency, microphthalmos, abnormal electroencephalograms, and postnatal growth deficiency. Unfortunately the authors did not indicate how often the same syndrome occurred in the other 907 cases. The publication prompted Fraser and Skelton, ${ }^{23}$ however, to look at data amassed over 25 years in the Montreal Children's Hospital. Out of 305 cases of abnormality, 55 of the mothers had been noted to have had a febrile episode, $36(11 \%)$ during the first five months of gestation. Only seven of the affected children had microphthalmia, but five $(71 \%)$ of these had a history of maternal febrile illness in the first half of gestation. This study got over the problem of biased recall by comparing answers of all mothers of infants with defects with one another. Though on its own neither study provides sufficient evidence to implicate a febrile illness, in combination they imply that this factor is of importance in the genesis of a rare syndrome, one of the distinguishing features being microphthalmia.

This conclusion does not imply that the lesion develops as a result of maternal hyperthermia: many other factors may be relevant, such as a direct effect of the pathogen responsible, the effect of drugs given, or maternal anorexia during the episode. If hyperthermia were the teratogen we should expect to find a relatively high prevalence of the syndrome among populations where saunas are taken frequently. In Finland, however, the incidence of microphthalmia appears to be only about 1 in $6000,{ }^{24}$ certainly no greater than that found in the United States by the collaborative project ( 1 in 3000). ${ }^{25}$ There is therefore still no convincing evidence to implicate hyperthermia per se as a human teratogen.

${ }^{1}$ Edwards, M J, Archives of Pathology, 1967, 84, 42.

2 Smith, L E W, Archives of Pathology, 1939, 28, 422.

${ }^{3}$ Lecyk, M, Experientia, 1966, 22, 254.

${ }^{4}$ Skreb, N, and Frank, Z, fournal of Embryology and Experimental Morphology, 1963, 11, 445.

5 Hartley, W J, Alexander, G, and Edward, M J, Teratology, 1974, 9, 299.

${ }^{6}$ Poswillo, D, et al, Annals of the Royal College of Surgeons of England, 1974, 55, 171.

7 Sohar, E, et al, Israel fournal of Medical Science, 1976, 12, 1275.

8 De Lee, J B (in discussion), in Murphy, D P, American fournal of Obstetrics and Gynecology, 1937, 34, 890.

${ }^{9}$ Hewitt, D, American fournal of Public Health, 1962, 52, 1676.

${ }^{10}$ Babbott, J G, and Ingalls, T H, American fournal of Public Health, 1962 $\mathbf{5 2}, 2009$.

${ }^{11}$ Doll, R, Hill, A B, and Sakula, J, British Fournal of Preventive and Social Medicine, 1960, 14, 167

12 Leck, I, British fournal of Preventive and Social Medicine, 1963, 17, 70.

${ }_{13}$ Leck, I, HSMHA Health Reports, 1971, 86, 265.

${ }_{14}$ Hakosalo, J, and Saxén, L, Lancet, 1971, 2, 1346.

15 Hardy, J M B, et al, American fournal of Public Health, 1961, 51, 1182.

16 Walker, W M, and McKee, A P, Obstetrics and Gynecology, 1959, 13, 394

17 Wilson, M G, and Stein, A M, fournal of the American Medical Association 1969, 210, 336.

18 Coffey, V P, and Jessop, W J E, Lancet, 1963, 1, 748. 